\title{
Multi-Layer Wireless Perception and Recognition Technology Based on Internet of Things
}

\author{
https://doi.org/10.3991/ijoe.v15i01.9776 \\ Er-hua Sun $\left({ }^{\bowtie}\right)$, Yu Chen \\ Chongqing Real Estate Career Academy, Chongqing, China \\ erhuasun38192@21cn.com
}

\begin{abstract}
To study the multi-layered wireless sensation and recognition technology of the Internet of Things, the background and research status at home and abroad were summarized. An IoT system architecture based on a multi-layer multi-reader passive network and a technology for simultaneous simulation of multiple network standards based on $\mathrm{OMNeT}++$ were proposed. The proportion of the time consumption of each communication phase in the entire communication process was analyzed in detail. A variety of strategies for shortening the system delay were proposed. At the same time, multiple network standard protocol stacks were divided and modeled. Dual-layer networks based on IEEE 802.11 and IEEE 802.15.4a were verified as examples. The results showed that the length of the hop sequence and the number of readers were optimized. Therefore, this method is effective.
\end{abstract}

Key Words-Internet of Things, wireless perception, recognition technology, multi-level

\section{Introduction}

The Internet of Things is an intelligent network that connects all objects together. Technologies and equipment such as radio frequency identification, wireless communication, real-time positioning, video processing, and sensing are used to enable any intelligent object to communicate information through a network. Physical objects are seamlessly integrated into the information network. The purpose is to connect every item to the network, which is convenient for management and identification. The Internet of Things is built using a variety of network technologies. One of the very important technologies is the RFID tag technology. Based on the RFID system, network technology, sensor technology, database technology, and middleware technology are integrated. The construction of a huge network consisting of a large number of networked readers and mobile tags that is larger than the Internet has become a trend in the development of the Internet of Things. In this network, the system can identify, locate, track, monitor and trigger corresponding events automatically and in real time.

The Internet of Things is widely used, especially in the areas of transportation, environmental protection and energy conservation, government agencies, industrial 
supervision, global security, home security, and healthcare. The Internet of things will improve the efficiency of the business process. Other applications include material handling and logistics, warehousing, tracking of products, management of data, reduction of production costs, acceleration of asset flow, anti-counterfeiting, and reduction of production errors. The defective product was immediately recalled. Waste is effectively recycled and utilized. The safety of drug prescriptions is enhanced. Food safety and quality are improved. In addition, the smart technology of the Internet of Things is integrated. For example, robots and wearable computers can make everyday objects a device for thinking and communicating. For example, an alarm may be issued when the pressure of a car tire is too low or if it is frozen on the road.

A multi-layer wireless sensing and identification system for the Internet of Things is built. Four key issues in the design and application of the Internet of Things are discussed. It includes protocol design, simulation platform and simulation method for physical layer and media access control layer, design of sensing nodes and network services, and design of video sensor codec hardware accelerator. Theoretical analysis, simulation methods, node design, and system implementation are deeply studied.

\section{State of the Art}

RFID is an automatic identification technology, which was proposed in 1948. Researchers at Los Alamos National Laboratory demonstrated passive passive tags and semi-active tags. This is an early RFID tag that uses reflected energy modulation. Liu et al. [1] pointed out that the operating frequency of this portable system is 915 $\mathrm{MHz}$ and 12-bit tags are used. This reflective modulation technique is widely used in today's UHF RFID and microwave RFID tags.

Tham [2] pointed out that MIT's Auto-ID Lab brought RFID manufacturers, researchers, and users together to jointly develop RFID standards, conduct related research, and share information for supply chain applications. EPC Global has been working on RFID standards for this application area. Li et al. [3] pointed out that the international organization for standardization has also made positive actions for the establishment of RFID standards in a large number of applications.

Mrissa et al. [4] defined the air interface protocol of the first generation of tags. This is the well-known Class 0 and Class 1 standard and has been commercialized. Baloch et al. [5] pointed out that through EPC Global's certification; a unified UHF RFID Type 1 Gen 2 air interface protocol was established. The EPC Type 1 Generation 2 standard incorporates the previous EPC Type 0 and Type 1 standards and the ISO 18000-6 Type A and Type B standards. After some revisions, it was adopted in 2006 as a Class 1 standard of ISO 18000-6. The first-generation standard contains descriptions of the UHF tags of Type 0 and Type 1. Alsheikh et al. [6] pointed out that the Type 0 agreement was initiated by Matrics Technology Systems (which was later acquired by Symbol Technology). The type 1 agreement was initiated by Alien Technology. Type 0 tags are defined as read-only devices by EPC Global, and Type 1 tags are defined as one-time programmable devices by EPC 
Global. In reality, the labels produced by Alien Technology can be duplicated. Geng and Dong [7] pointed out that Matrics/Symbol has issued 0+ products. This product uses the same protocol as the Type 0 product. In fact, it is completely rewritable [7]. In order to improve the first-generation standard, EPC introduced the Type 1 Generation 2 standard. Type 0 protocols have been abandoned. The second-generation standard has significantly improved performance over the first-generation standard. It includes faster and more flexible read and write speeds. The identification of tags is more reliable. Security is enhanced. With multiple readers, the robustness and scalability of performance are improved. Wu et al. [8] proposed a DFSA algorithm. The algorithm estimates the unrecognized tag by multiplying the number of conflicting slots in the current frame by 2.39 times the posterior probability value of the number of tags in each conflicting slot and uses this value to adjust the length of the next frame. This algorithm is very effective when the number of tags is very close to the frame length. However, when the difference between the number of tags and the frame length is large, the estimation error of the algorithm will increase significantly. A method using the distance between the observation and the expected vector is proposed to estimate the number of tags. At least two tags responded in the collision time slot, and the lower limit of the total number of tags was estimated as FS +2 Fc. Among them, Fs is the number of successful slots in the current frame, and $\mathrm{Fc}$ is the number of collision slots. Then, the algorithm uses Markov process to model the tag identification process, and gives a recommended dynamic frame length set. To obtain the minimum vector distance, the algorithm requires a lot of calculations.

\section{$3 \quad$ Methodology}

\subsection{Internet of Things based on passive RFID system}

The concept of the Internet of Things was first proposed in 1999 by the Auto-ID Center at the Massachusetts Institute of Technology (MIT). If everything from books to cars in daily life is equipped with sensor tags, they can be recognized and managed by computers. The Internet of Things will encode 50 to 100 trillion objects and track their movement. Different existing communication standards will be used to build the IoT infrastructure. The Internet of Things needs to use a multi-layered network structure to obtain information from target objects and deliver it to users.

Figure 1 shows an example of a multi-layer network architecture based on a passive RFID system. It mainly includes three components: server, reader and sensor tags. They form a three-tiered network. The upper layer network is used for communication between the server and the reader. The middle-level network is used for communication between readers. The underlying network is used for communication between readers and tags. In the upper network, the server acts as a control center for the reader/writer cluster through wired connections (such as USB, RS-232, Ethernet, etc.) or wireless connections (such as Zigbee, Wi-Fi, WiMAX, GSM/GPRS, etc.). A reader cluster can contain one or more readers. The computer can be used as a server to store and process the data sent by the reader. The server is 
also a gateway to an external network (Internet) and can provide network services. In an intermediate network, readers in a reader-writer cluster can implement selforganizing connections. The purpose of the intermediate network is to provide an effective cooperation mechanism between readers and handle the conflict between readers. In the underlay network, passive RFID standards, such as the EPC Class 1 Generation 2 standard, are used to reduce the conflict between tags and to effectively collect and preprocess the tag's sensory information.

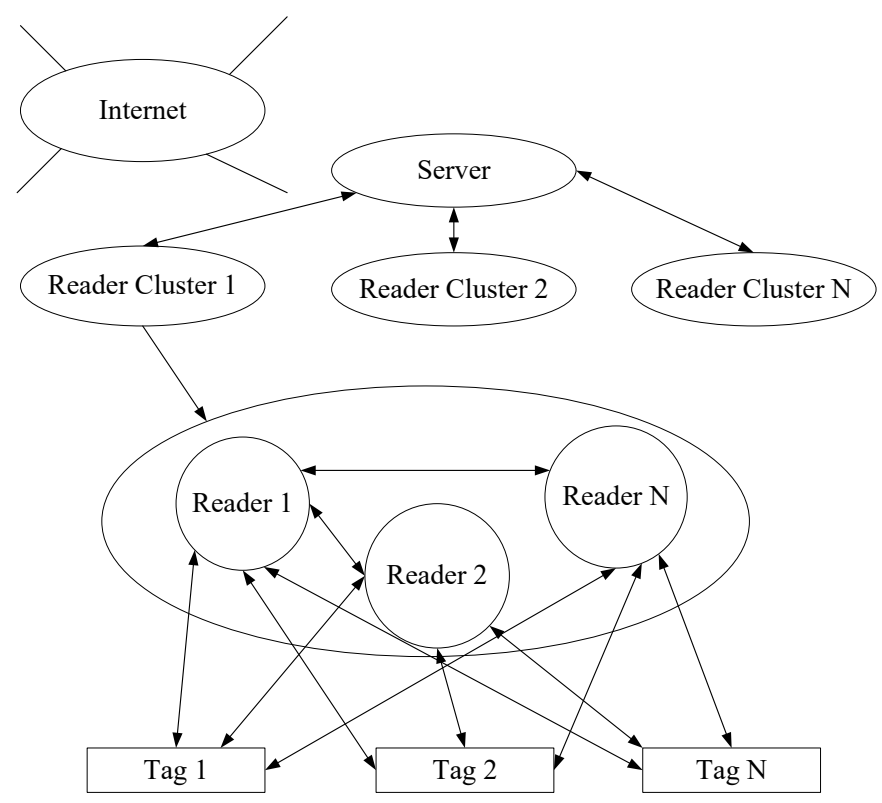

Fig. 1. Multi-layer Internet of Things based on passive RFID system

\subsection{Principle of reflection modulation}

The RFID system using reflected energy is essentially a radar system. The reader provides energy for two-way communication. The tag does not have an active transmitter. It transmits its ID and other data by modulating the energy reflected from the tag antenna. The energy emitted by the reader is also used to increase the necessary operating voltage for the tag's circuitry. The tag can change the amplitude or phase of the reflected signal by changing the real or imaginary part of the chip impedance.

The reader antenna and the tag antenna have the same polarity. When the distance between the reader and the tag is $r$, the density $S$ of the radiated power received by the tag can be obtained by calculating the quotient of the transmitted power and the sphere surface area, as shown in the following equation:

$$
\mathrm{S}=\frac{P_{E I R P}}{4 \pi r^{2}}=\frac{P_{1} G_{1}}{4 \pi r^{2}}
$$


In the formula, $\mathrm{P}_{\text {EIRP }}$ is the effective isotropic radiation power. $\mathrm{P}_{1}$ is the radiated power of the reader antenna. $G_{1}$ is the gain of the reader and writer antenna. Figure 2 shows a simplified model of RFID system using reflection energy.

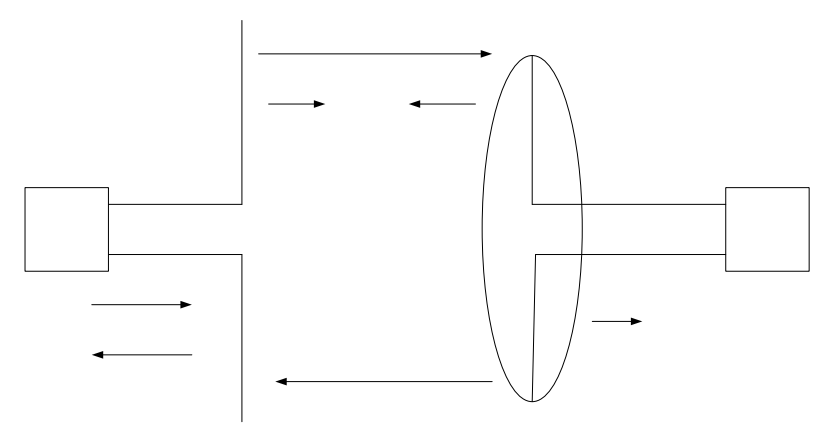

Fig. 2. Simplified model of RFID system using reflected energy

Through analysis, it can be known that the reflected power of the tag received by the reader is inversely proportional to the distance between the reader and the tag. It is related to the directionality of the reader antenna and the tag antenna, and the matching condition of the tag antenna and the chip. These characteristics of the reflection modulation make passive RFID systems based on reflection modulation very sensitive to the distance between the reader and the tag, and are susceptible to multipath effects and multi-user interference. In some cases, the identification of tags is very difficult.

\subsection{A brief introduction to the simulation platform}

The construction of the Internet of Things needs to make existing communication protocols compatible with each other. The new multi-layer wireless network communication protocol should be designed. Intelligent node hardware and firmware design, middleware development and field testing can truly reflect the performance of the protocol. However, the design of hardware, the construction of networks, and the testing of experiments will consume a lot of time and money, and the Internet of Things will grow rapidly in size and complexity. Accurate simulation of wireless network protocols has many advantages in terms of time and cost. At present, there are many kinds of network simulation tools, including NS-2, OPNET, OMNeT++, G1oMoSim, J-Sim, QualNet and so on. However, different network simulation tools have their own deficiencies. For example, the model is too simple, and user customization is not supported. The transplantation of existing protocol modules is difficult to obtain and the price is very high. The network simulation tool for the project is very important for developers. The three most commonly used network simulation tools, NS-2, OPNET and $\mathrm{OMNeT}++$, are mainly introduced and compared.

NS-2 lacks user customization support without graphical interfaces or layered models. Both OPNET and OMNeT++ support user-defined packet structures, 
graphical interfaces, and arbitrary depth layered models. OMNeT++ also supports user-customized and parameterized topologies, while OPNET only supports fixed topologies. OMNeT++ is much more flexible and extensible than NS-2, and it is better than OPNET. OMNeT++ supports graphical runtime environment and target detection, which makes tracing and debugging OMNeT++ programs simpler and faster than NS-2 and OPNET. However, network simulation tools often assume that the propagation of wireless signals is only a function of distance. Such an overly simple model makes the simulation of certain applications inaccurate. In contrast, MATLAB has an accurate channel model and a large number of powerful functions and models. However, MATLAB is not suitable for task scheduling. Table 1 compares the respective characteristics of these four tools in different aspects:

Table 1. Comparison of simulation tools

\begin{tabular}{|c|c|c|c|c|}
\hline Tool & NS-2 & OPNET & OMNeT++ & MATLAB \\
\hline $\begin{array}{l}\text { Interface } \\
\text { language }\end{array}$ & $\mathrm{C}++/ \mathrm{OTCL}(\mathrm{IL})$ & $\mathrm{C} / \mathrm{C}++(\mathrm{CL})$ & $\mathrm{C}++/ \mathrm{NED}(\mathrm{CL})$ & MATLAB(IL) \\
\hline $\begin{array}{l}\text { Support } \\
\text { module }\end{array}$ & TM,MANET,Zigbee & TM,MANET,WSN & $\begin{array}{l}\text { TM,MANET,WSN,IEEE } \\
802.15 .4\end{array}$ & $\begin{array}{l}\text { Accurate } \\
\text { channel } \\
\text { Harbin } \\
\text { Physical layer } \\
\text { model, DSP, } \\
\text { matrix } \\
\text { operation }\end{array}$ \\
\hline $\begin{array}{l}\text { Dynamic } \\
\text { module }\end{array}$ & Support & Support & Support & Nonsupport \\
\hline Parallelism & Nonsupport & Support & MPI/PVM & Support \\
\hline Extensibility & Small & Secondary & Large & Large \\
\hline $\begin{array}{l}\text { Tracking / } \\
\text { debugging }\end{array}$ & $\begin{array}{l}\text { Very limited visual } \\
\text { support, consuming } \\
\text { memory }\end{array}$ & $\begin{array}{l}\text { Command line } \\
\text { debugging }\end{array}$ & $\begin{array}{l}\text { Module output window, } \\
\text { target detection, graphic } \\
\text { running environment }\end{array}$ & $\begin{array}{l}\text { Intuitionistic } \\
\text { parameter } \\
\text { detection, } \\
\text { time } \\
\text { consuming }\end{array}$ \\
\hline $\begin{array}{l}\text { Authorization } \\
\text { license }\end{array}$ & Open Source & Expensive & Open Source & Low \\
\hline
\end{tabular}

\subsection{Network standards and the composition of the Internet of Things}

The Internet of Things will be a Heterogeneous Network. It consists of mobile communication networks, the Internet, Wi-Fi, Bluetooth, Global Position System (GPS), and satellite communication networks. A large number of heterogeneous wireless terminal devices will be densely distributed in every corner of life. The Internet of Things needs to integrate these heterogeneous networks. The function is more complicated. The scale is even bigger. Real-time web services need to be provided.

Passive UHF RFID (such as the EPC Class-1 Generation-2 protocol) and wireless sensor networks based on the IEEE 802.15.4 standard (such as the Zigbee protocol) are considered to be the two cornerstones that make up the Internet of Things. However, mobile communications and WLAN are overlapped with UHF and IEEE 802.15.4 respectively. Passive UHF RFID tags typically operate at $860-960 \mathrm{MHz}$. The GSM900 uplink frequency band in China Mobile's frequency plan is $890-915 \mathrm{MHz}$, 
and the downlink is $935-960 \mathrm{MHz}$. The band that is applicable to the world in the IEEE 802.15.4 protocol is $2400-2483.5 \mathrm{MHz}$, which has 16 channels. The working frequency of WLAN IEEE $802.11 \mathrm{~b} / \mathrm{g} / \mathrm{n}$ includes the scope of $2.4 \mathrm{GHz}$. When such multiple network standards coexist, there may be serious interference between them.

Therefore, for the simulation of the Internet of Things, it is necessary to support simultaneous simulation of multiple wireless network standards in one simulation instance. The characteristics of various network standards are reflected and the interference between them is considered. Although existing network simulation tools provide a variety of network standard modules, they do not give a method for how to simulate these modules in a simulation instance at the same time, and such simulation examples are lacking.

A method for simultaneous simulation of multiple network standards based on $\mathrm{OMNeT}++$ is proposed. For a given IoT application, network standards need to be considered first. The protocol stack is divided into these network standards respectively. The simulation module of each layer of each protocol stack is built. Then, the types of heterogeneous nodes in the Internet of Things and the network standards for each node are considered. Therefore, according to each protocol stack module, a node's simulation model is built, and an interface module between each standard is designed.

\section{Result Analysis and Discussion}

\subsection{Multilevel asymmetric network structure}

In passive RFID systems, the number of readers is much smaller than the number of tags. The forward connection from the reader to the tag and the reverse connection from the tag to the reader are extremely asymmetric in the amount of data transferred. Reverse link has higher requirements on data rate. The EPC Class 1 Generation 2 standard uses UHF for both forward and reverse connections, and the data rates are not much different. In contrast, to better adapt to this asymmetric structure of passive RFID systems, an asymmetric wireless connection strategy between the reader and the tag is proposed. That is, UHF is used as the forward connection, and TH-PPM and IRUWB are used as the reverse connection. UWB can achieve a data rate of $10 \mathrm{Mbps}$, which can well meet the requirements of large amount of reverse connection data. The UWB transmitter has a simple structure and low power consumption. However, the UWB receiver has high complexity and large power consumption, which makes the UWB receiver unable to work through remote wireless power supply and cannot be applied to passive tags. Therefore, UWB can only be used in reverse connection. The system needs this asymmetric structure. Because asymmetrical connections are used, there is no frequency interference between the forward connection and the backward connection. This allows the forward and backward connections to partially overlap in time and avoids interference from the reader to the reader and accelerate the process of tag identification. Moreover, UWB is not easy to be intercepted, which improves the confidentiality and security of information for RFID systems. With UWB signals, 
millimeter-level positioning accuracy is achieved. This is of great significance to the construction of the Internet of Things. Orthogonal TH-PPM IR-UWB is applied to the reverse connection, which can effectively reduce the conflict between tags. Different readers may successfully receive information from different tags in the same time slot, which can further speed up the tag identification process.

Based on this asymmetric connection, a multi-level and asymmetrical network structure is proposed. The network contains servers, readers and passive tags. The server contains a Wi-Fi network card. The reader includes a Wi-Fi network card, an UHF transceiver, and an orthogonal TH-PPM IR-UWB receiver. The passive tag contains an UHF receiver and a quadrature TH-PPM IR-UWB transmitter. They form a three-tier wireless network, namely the upper network, the middle network, and the underlying network.

The upper network is used for communication between the server and the reader. It is a star network. The server communicates with the reader through the Wi-Fi connection based on the IEEE 802.11 protocol and schedules the reader through the network synchronization. In each inventory cycle, a primary reader is selected. The server processes the data information sent by the reader at the end of each inventory cycle. For example, the information of the tag is recorded in the database, and the position of the tag is calculated. The middle-level network is used for communication between readers. In each inventory cycle, the master reader uses the $860-960 \mathrm{MHz}$ UHF connection to send inquiries to tags and other readers. Other readers act as auxiliary readers during this inventory cycle. They change the status according to the instructions of the main reader and record the response information of the tag and respond to the tag by way of competition. The underlying network is used for communication between readers and tags. It has a many-to-many structure. The tag obtains energy from the UHF signal sent by the reader and the electromagnetic waves in the surrounding environment. It changes state and responds according to the reader's instructions. Multilevel asymmetrical passive network structure is shown in Figure 3. 


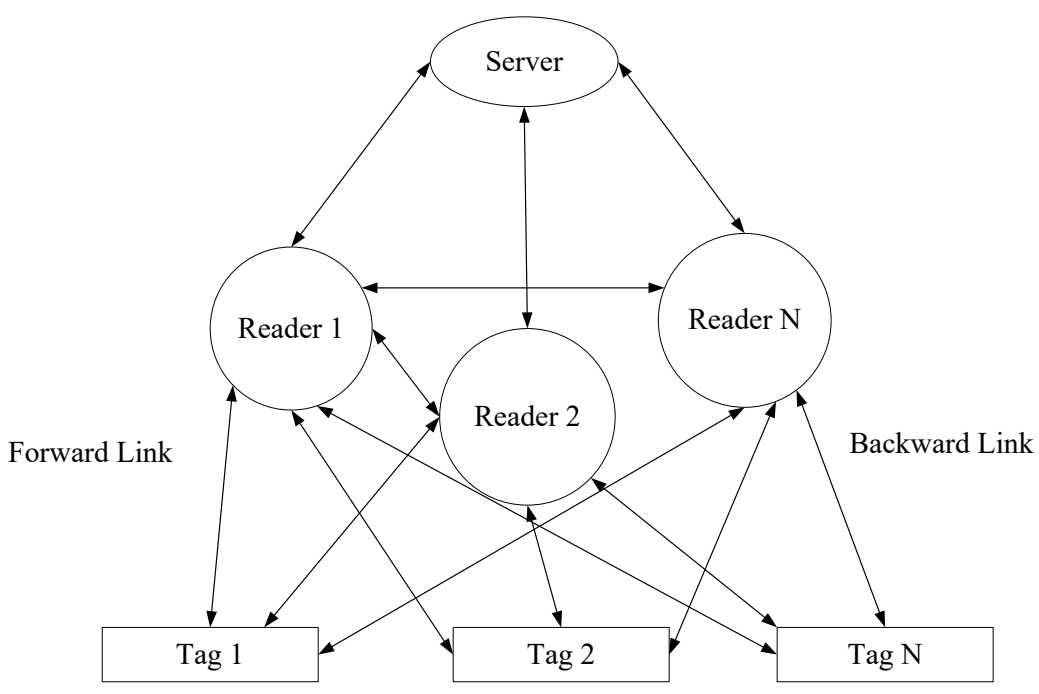

Fig. 3. Multilevel asymmetrical passive network structure

\subsection{Performance analysis and comparison}

To verify its good performance, CDMA-based multi-reader passive RFID systems, EPC Class-1 Generation-2 systems and multi-user detection-based systems were compared in dense environments. The readers in the three systems work on only one frequency band and do not use frequency hopping. The reason is that in this application environment, frequency hopping will cause the reader to conflict with the tag. Many RFID applications need to know the location of the tag, which usually requires multiple readers to monitor the same channel at the same time. Therefore, all three systems use multiple readers. They all use the same tag clock frequency. Both the proposed system and the multi-user detection-based system only use the Gold code for the RN 16 information. EPC Class-Z Generation-2 systems and systems based on multi-user detection both use network synchronization to avoid reader-to-tag conflicts and reader-writer-to-reader conflicts.

With different numbers of tags, the system uses 8 readers. The minimum value of the multi-user detection system delay expectation and the EPC Class- 1 Generation-2 system and the proposed system are simulated. The latency of the three systems increases linearly with the number of tags. After using the Gold code, the RN 16 data is 7 times longer than the EPC Class-1 Generation-2 system. However, the recognition rates of the proposed system and the multi-user detection system are 1.6 and 1.8 times that of the EPC Class-1 Generation-2 system, respectively. With the Gold code, tag-to-tag conflicts are greatly reduced. The total number of time slots used by the proposed system and the multi-user detection system is one-fifth and oneninth, respectively, of the EPC Class-1 Generation-2 system.

Compared with the proposed system, the multi-user detection system has better system performance under ideal conditions. However, the multi-user detection system 
will be seriously affected by the received signal power, phase shift, and channel noise in real applications. Moreover, multi-user detection systems often need to know the number of response tags in a time slot, but this number is unpredictable in advance. In this way, the multi-user detection system needs to recalculate the inverse matrix of the correlation matrix or eliminate the interference with multiple stages in each time slot. These methods increase the complexity of the reader and the time of processing the signal. On the contrary, in the case of a certain number of readers and tags, the proposed system is lower in cost than the multi-user detection system and is more suitable for RF1D systems. Comparison of system performance is shown in Figure 4.

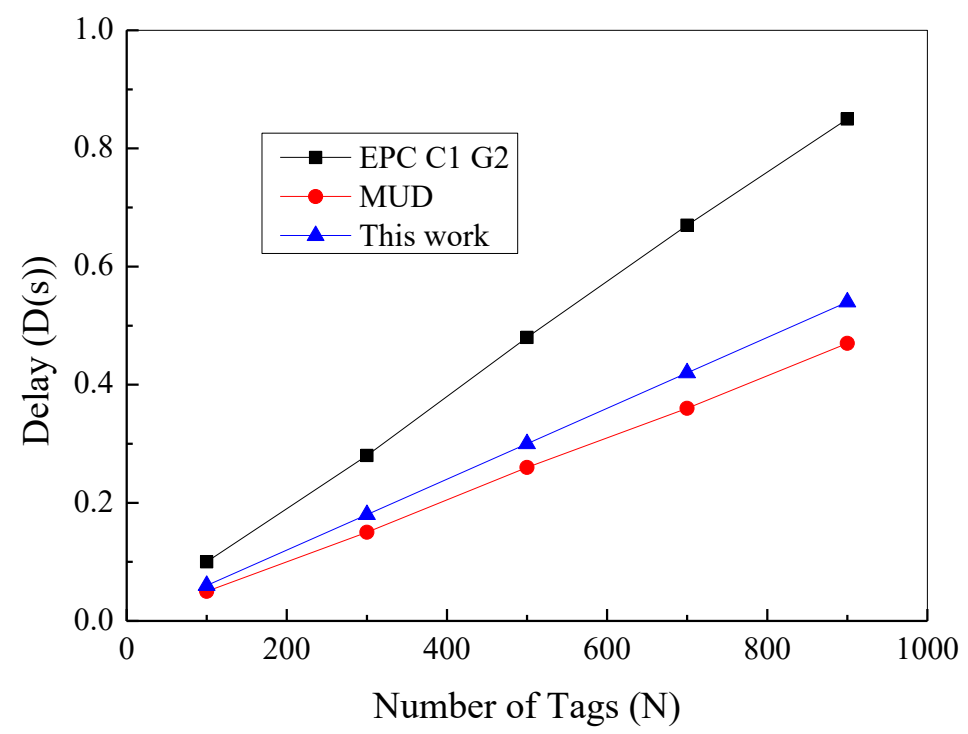

Fig. 4. Comparison of system performance

\subsection{Simulation example based on COSMO}

Currently, in order to meet customer requirements and reduce costs, retailers manage inventory automatically. However, due to the wrong placement of items, the accuracy of the inventory of the world-renowned retailers is only $51 \%$. RFID positioning technology can effectively reduce the possibility of misplaced items, and can also help operators quickly find the wrong items. Compared with active RFID positioning, passive RFID positioning has the advantages of low cost, no battery, and low maintenance cost, which makes passive RFID positioning more attractive for inventory management.

Simulation examples of passive RFID positioning based on COSMO are given. EPC Class-1 Generation-2 UHF passive RFID network is used to locate items in supermarkets. To demonstrate the power of COSMO, COSMO and other network simulation tools were compared in the same passive RFID network scenario. 
The positioning error for a stationary tag is $0.8 \mathrm{~m}$, which hardly varies with the number of tags. However, when the number of tags increases from 100 to 900 , the positioning error for moving tags will gradually increase. The proposed multi-layer passive RFID network has a positioning error of $0.8 \mathrm{~m}$ for stationary tags. A mobile tag that tracks S00 speeds of no more than $3 \mathrm{~m} / \mathrm{s}$ with a positioning error of no more than $1 \mathrm{~m}$ is implemented. When the speed of moving the label is faster, the positioning error will increase accordingly. When the speed of the moving label exceeds $3 \mathrm{~m} / \mathrm{s}$, the positioning error will increase linearly with the label speed. Positioning error (maximum speed of label $3 \mathrm{~m} / \mathrm{s}$ ) is shown in Figure 5.

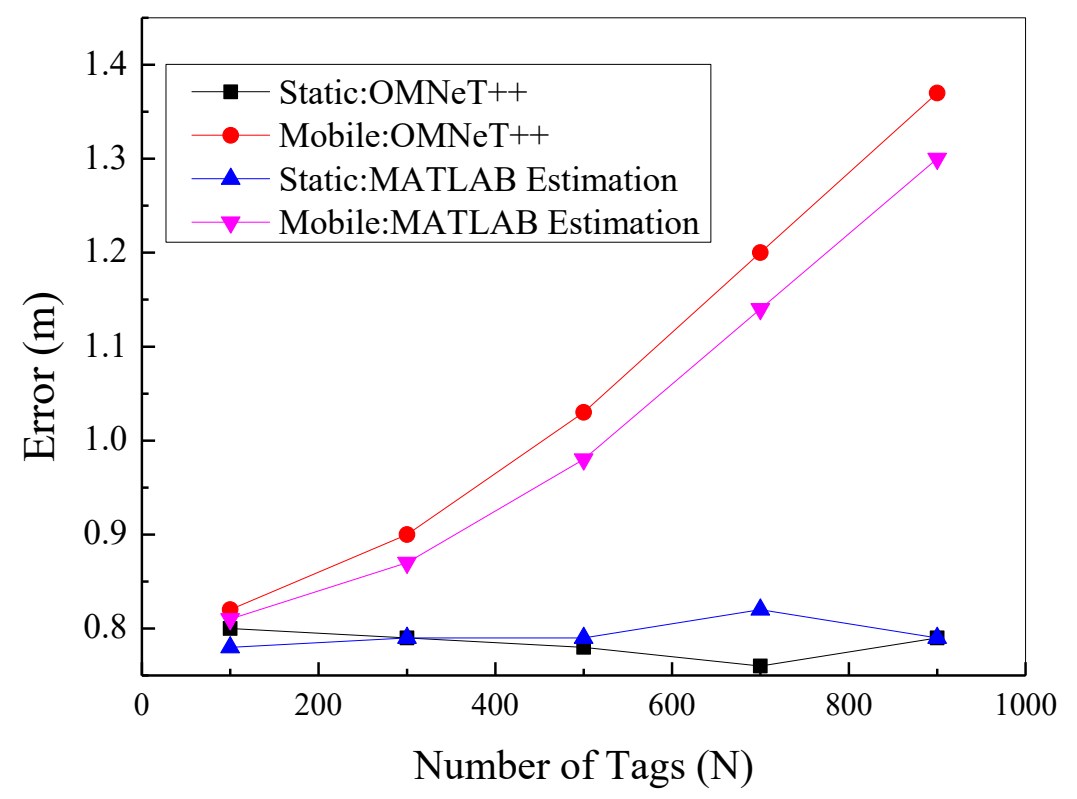

Fig. 5. Positioning error (maximum speed of label $3 \mathrm{~m} / \mathrm{s}$ )

Simulation results in MATLAB can be used to verify the results of the cosimulation. MATLAB positioning errors and processing delays for a tag can be used to estimate the positioning error of multiple tags in a co-simulation. In a read cycle, the time at which the tag is identified is evenly distributed between the processing delay minimum and maximum values. Then, this tag is read 1000 times and the average of the positioning error is calculated. Since this tag may be identified in a different time slot in a read cycle, the average of its positioning error can be used as an approximate estimate in the case of multiple tags. As shown in Figure 5, for stationary and moving tags, the MATLAB estimates all agree with the results of the co-simulation. 


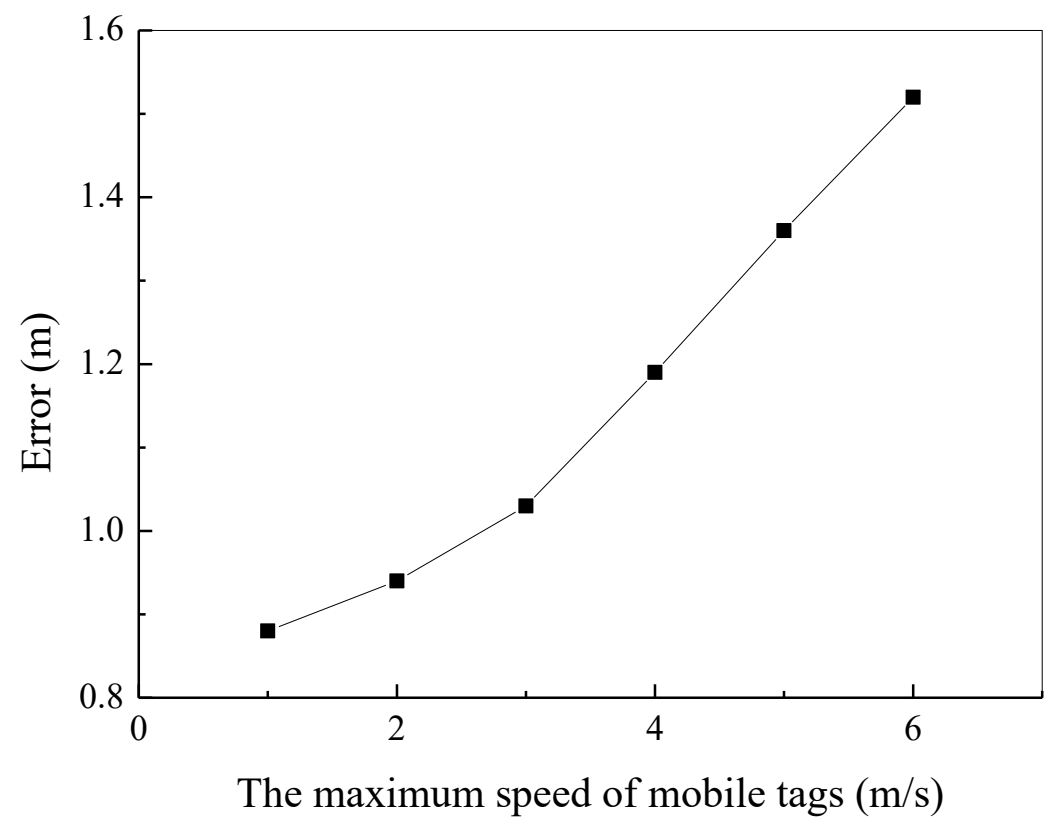

Fig. 6. Positioning error for 500 mobile tags

\section{Conclusion}

The passive UHF RFID standard overview, the difference between the reflection modulation in the physical layer and the simulation platform was introduced. According to the evolution of the passive UHF RFID standard, the anti-collision algorithm of the tag evolved from the algorithm based on the tree structure to the ALOHA algorithm based on the dynamic frame slot. Miller's modulation method was proposed, and the frequency hopping and dense reader modes of operation were added. In addition, a protocol design and optimization method based on CDMA multiple access is proposed, and a multi-reader passive UHF RFID system for dense environments is designed. The cost of the proposed reader is slightly higher than that of the reader in the EPC Class-1 Generation-2 system, but it is much lower than the cost of the reader detected by the multiuser.

Using examples, the results show that a system of 8 readers and 9 7-bit long Gold codes can achieve the best compromise between recognition rate and cost. With this configuration, the proposed system can achieve a recognition rate of 1670 tags per second. The number of readers is doubled. When using 16 readers, only the recognition rate can be increased by 3.6. With the same configuration, the proposed system is $58 \%$ faster than the EPC Class-1 Generation-2 system. Compared with other network simulation tools, when COSMO is used to build the network, the workload is reduced by half, and the simulation results can be verified by COSMO itself. 


\section{References}

[1] Liu, X., Wang, P., Lan, Z., \& Shao, B. (2015). Biological characteristic online identification technique over 5G network. IEEE Wireless Communications, 22(6): 84-90 https://doi.org/10.1109/MWC.2015.7368828

[2] Tham, J. C. K. (2018). Interactivity in an age of immersive media: Seven dimensions for wearable technology, internet of things, and technical communication. Technical Communication, 65(1): 46-65

[3] Li, S., Chen, W., Hu, J., \& Hu, J. (2018). ASPIE: A Framework for Active Sensing and Processing of Complex Events in the Internet of Manufacturing Things. Sustainability, 10(3): 692 https://doi.org/10.3390/su10030692

[4] Mrissa, M., Médini, L., Jamont, J. P., Le Sommer, N., \& Laplace, J. (2015). An avatar architecture for the web of things. IEEE Internet Computing, 19(2): 30-38 https://doi.org/10.1109/MIC.2015.19

[5] Baloch, Z., Shaikh, F. K., \& Unar, M. A. (2018). A context-aware data fusion approach for health-IoT. International Journal of Information Technology, 10(3): 241-245 https://doi.org/10.1007/s41870-018-0116-1

[6] Alsheikh, M. A., Niyato, D., Lin, S., Tan, H. P., \& Han, Z. (2016). Mobile big data analytics using deep learning and apache spark. IEEE network, 30(3): 22-29 https://doi.org/10.1109/MNET.2016.7474340

[7] Geng, L., \& Dong, T. (2017). An Agricultural Monitoring System Based on Wireless Sensor and Depth Learning Algorithm. International Journal of Online Engineering (iJOE), 13(12): 127-137 https://doi.org/10.3991/ijoe.v13i12.7885

[8] Wu, Q., Ding, G., Xu, Y., Feng, S., Du, Z., Wang, J., \& Long, K. (2014). Cognitive internet of things: a new paradigm beyond connection. IEEE Internet of Things Journal, 1(2): 129-143 https://doi.org/10.1109/JIOT.2014.2311513

\section{$7 \quad$ Authors}

Er-hua Sun and Yu Chen are Researchers of Chongqing Real Estate Career Academy, Chongqing, China. Their research interests include Multi-layer Wireless Perception and Recognition Technology.

Article submitted 20 October 2018. Resubmitted 24 November 2018. Final acceptance 12 December 2018. Final version published as submitted by the authors. 